\title{
Caminhada em Ambiente Aquático e Terrestre: Revisão de Literatura Sobre a Comparação das Respostas Neuromusculares e Cardiorrespiratórias
}

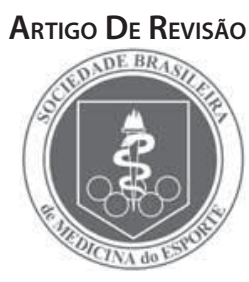

\section{Walking on Land and in Water: a Review Study About the Comparison of Neuromuscular and Cardiorespiratory Responses}

Eduardo Marczwski da Silva'

Luiz Fernando Martins Kruel ${ }^{1}$

1. Universidade Federal do Rio Grande do Sul. Escola de Educação Física. Laboratório de Pesquisa no Exercício - Lapex. Porto Alegre, RS - Brasil.

Endereço para correspondência: Eduardo Marczwski da Silva. Rua Felizardo, 750. Jardim Botânico. Escola de Educação Física/LAPEX, sala 208 - 90690-200 - Porto Alegre, RS - Brasil.

E-mail:

eduardomarczwski@yahoo.com.br

Submetido em 02/04/2008

Versão final recebida em 16/06/2008 Aceito em 05/07/2008

\begin{abstract}
RESUMO
O objetivo deste estudo foi realizar uma revisão de literatura sobre a comparação das respostas neuromusculares e cardiorrespiratórias durante a caminhada em meio aquático e em meio terrestre. As respostas foram apresentadas para a caminhada em piscina rasa e para a caminhada em piscina funda. Em relação à caminhada em piscina rasa, as respostas neuromusculares (sinal EMG) e cardiorrespiratórias $\left(F C\right.$ e $\left.\mathrm{VO}_{2}\right)$ são muito dependentes da velocidade do exercício. As respostas neuromusculares podem ser menores no meio aquático quando a velocidade da caminhada é menor nesse ambiente. Por outro lado, quando o exercício é realizado com velocidades similares, a atividade dos músculos propulsores pode ser superior durante a caminhada aquática. Da mesma forma, respostas cardiorrespiratórias maiores são registradas no exercício aquático quando velocidades similares de caminhada são utilizadas. Contudo, com velocidades menores, essas respostas são semelhantes ou menores que as encontradas em meio terrestre. No que diz respeito à caminhada em piscina funda, as respostas neuromusculares diferem daquelas encontradas durante a caminhada em meio terrestre devido à ausência das forças de reação com o solo. Essa característica possivelmente modifica os músculos envolvidos na produção de força propulsiva nesse exercício. Além do mais, durante a caminhada em piscina funda, as respostas cardiorrespiratórias parecem sempre mais baixas devido à grande redução no peso hidrostático e à menor atividade dos músculos posturais e pela utilização do cinturão flutuador nessa modalidade.
\end{abstract}

Palavras-chave: caminhada em piscina rasa, caminhada em piscina funda, respostas fisiológicas.

\begin{abstract}
The purpose of this review was to analyze neuromuscular and cardiorespiratory responses during walking in water and on dry land. The responses were presented during walking in shallow and in deep water. During walking in shallow water, neuromuscular (EMG signal) and cardiorespiratory ( $\mathrm{HR}$ and $\left.\mathrm{VO}_{2}\right)$ responses are very dependant on the exercise speed. Neuromuscular responses can be lower in aquatic environment when speed is lower. On other hand, when the exercise is performed in similar speeds, propulsive muscles activity can be higher walking in shallow water. Similary, higher cardiorespiratory responses are registered when similar speeds are used during the aquatic exercise. However, when it is performed at lower speeds, cardiorespiratory responses are similar or lower than the ones found on land. Concerning walking in deep water, neuromuscular responses are different from walking on land due to the absence of the ground reaction forces. This characteristic probably modifies the muscles involved in propulsive forces production during the exercise. Moreover, cardiorespiratory responses always seem to be lower than those during walking on land due to the effect of hidrostatic pressure as well as the lower posture muscles activity observed by the use of the floating belt in this modality.
\end{abstract}

Keywords: walking in shallow water, walking in deep water, physiological responses.

\section{INTRODUÇÃO}

Recentemente, o ambiente aquático tem sido utilizado para a prática de diversas atividades físicas além da natação, como a hidroginástica, a corrida e a caminhada ${ }^{(1-4)}$. Tais atividades são recomendadas em função dos benefícios metabólicos, cardiorrespiratórios, na composição corporal e na força muscular dos praticantes ${ }^{(5-9)}$. Esses benefícios são similares aos encontrados no exercício terrestre, com a vantagem da redução nas forças de compressão sobre o sistema musculoesquelético dos indivíduos durante o exercício aquático(3,10-13).

O exercício de caminhada em meio aquático pode ser caracteriza- do por dois tipos: a caminhada em piscina rasa ${ }^{(12,14)}$ e a caminhada em piscina funda ${ }^{(15,16)}$. Independentemente do exercício de caminhada realizado, a prática dessa atividade no meio aquático parece gerar diferentes respostas neuromusculares e cardiorrespiratórias comparadas com a caminhada em meio terrestre. Tais respostas estão associadas a algumas características físicas do ambiente aquático (força de empuxo, densidade, pressão hidrostática e termodinâmica) que o diferenciam do $\operatorname{ar}^{(3,6,8)}$.

Do ponto de vista neuromuscular (atividade eletromiográfica) e cardiorrespiratório (freqüência cardíaca e consumo de oxigênio), as diferentes respostas encontradas no ambiente aquático devem-se à 
maneira como as características desse ambiente influenciam fisicamente e fisiologicamente o corpo humano em imersão, durante o exercício. Essa influência é fundamental para a determinação da intensidade do exercício através da resistência oferecida ao movimento(17-20). Assim, as respostas neuromusculares e cardiorrespiratórias geradas durante a caminhada no meio líquido podem diferir daquelas encontradas em meio terrestre ${ }^{(21-25)}$, dependendo do tipo de exercício realizado (piscina rasa ou funda) e da maneira como a resistência do ambiente aquático $\left(R=0,5 \cdot d \cdot A \cdot v^{2} \cdot C_{d}\right)$ é empregada ao exercício ${ }^{(12,14,17-20,25)}$.

Nessa perspectiva, o conhecimento das situações que levam a diferentes respostas neuromusculares e cardiorrespiratórias é de fundamental importância para que a prescrição da caminhada nesse ambiente seja de acordo com os objetivos propostos ${ }^{(1,14,22)}$. Dessa forma, 0 objetivo do presente estudo foi realizar uma revisão de literatura sobre a comparação das respostas cardiorrespiratórias e neuromusculares durante a caminhada em meio aquático, de acordo com o tipo de caminhada e de resistência oferecida a esse exercício, e a caminhada em meio terrestre.

\section{METODOLOGIA}

Para a realização da presente revisão, a grande maioria das referências foi retirada das bases de dados Pubmed e Scopus, disponíveis na internet. Nessa perspectiva, foram buscados principalmente artigos atuais publicados a partir do ano 2000. Pela maior disponibilidade e facilidade na procura e na leitura, foram preferencialmente utilizados artigos publicados em língua portuguesa ou inglesa. Para isso, palavras-chave como "walking on land", "heart rate", "oxigen consumption", "electromyographic", "walking in shallow water", "walking in deep water", "cardiorespiratory and neuromuscular responses", "exercise", suas respectivas traduções em português, entre outras, foram combinadas de diversas maneiras. Referências anteriores ao ano 2000, citadas em muitos dos estudos atuais, também foram buscadas, diretamente por correspondência (e-mail) com os autores, ou ainda, em revistas disponíveis na biblioteca desta Universidade. Ainda, a dissertação de mestrado e a tese de doutorado apresentadas podem ser encontradas na biblioteca da Universidade onde foram realizadas. Por fim, as citações referentes a congressos da área podem ser encontradas nos respectivos anais desses congressos.

\section{RESPOSTAS NEUROMUSCULARES}

\section{Caminhada em piscina rasa}

Devido às dificuldades em coletar o sinal EMG no meio aquático e as contínuas investigações na tentativa de melhorar a utilização dessa técnica, é recente o interesse pelas respostas neuromusculares da caminhada nesse ambiente ${ }^{(12,23,26-29)}$. Durante a caminhada em piscina rasa, os autores têm manipulado a velocidade e o tipo de deslocamento frontal como os principais influenciadores da resistência imposta ao movimento dos indivíduos. Visto que esses fatores influenciam diretamente a intensidade do exercício, as respostas encontradas parecem extremamente dependentes dos mesmos.

Estudos comparativos com velocidade auto-selecionada e deslocamento frontal livre demonstram resultados semelhantes. As respostas neuromusculares analisadas a partir da atividade eletromiográfica (EMG), tanto de músculos posturais quanto de músculos propulsores, parece menor durante a caminhada em meio aquático comparada com a caminhada em meio terrestre quando apenas uma velocidade auto-selecionada de esforço (leve, moderada ou alta) é utilizada no exercício ${ }^{(12,13,21,28)}$. Isso se deve, possivelmente, ao fato de que para uma mesma velocidade auto-selecionada de esforço (moderado), a velocidade de deslocamento horizontal no meio aquático $(1,8 \mathrm{~km} / \mathrm{h})$ é sempre menor quando comparada com a do meio terrestre $(5 \mathrm{~km} / \mathrm{h})^{(12)}$.
Essa menor velocidade de deslocamento horizontal durante a caminhada aquática ocorre provavelmente pela necessidade de deslocar-se em ambiente de maior densidade ${ }^{(19,23,28,29)}$. Uma vez que a resistência imposta ao deslocamento no meio aquático aumenta ao quadrado em relação ao aumento da velocidade, a menor velocidade registrada no nesse ambiente justificaria a atividade neuromuscular diminuída ${ }^{(12,19)}$. Associada a menor velocidade de deslocamento horizontal está a redução do peso hidrostático como resultado da imersão, provocada pela presença da força empuxo ${ }^{(11)}$. Esse menor peso hidrostático pode resultar em menores forças propulsivas e de manutenção postural durante a caminhada no meio aquático(12).

Respostas similares para a ativação dos músculos propulsores da caminhada são encontradas em investigações com velocidade fixa (mesma intensidade fisiológica de exercício) e deslocamento frontal reduzido (esteira) ${ }^{(14,22,23,30,31)} \mathrm{em}$ músculos responsáveis pela propulsão do movimento. Vale salientar que para a obtenção de uma mesma intensidade fisiológica de esforço entre os meios, a velocidade do exercício terrestre precisa ser a do dobro da velocidade do exercício aquático (terra $=2,4 \mathrm{~km} / \mathrm{h}, 3,6 \mathrm{~km} / \mathrm{h}$ e $4,8 \mathrm{~km} / \mathrm{h}$ vs água $=1,2 \mathrm{~km} / \mathrm{h}$, $1,8 \mathrm{~km} / \mathrm{h}$ e $2,4 \mathrm{~km} / \mathrm{h})^{(25,30,31)}$. Da mesma forma, a menor atividade de músculos como o gastrocnêmio, o tibial anterior, vasto medial, o reto femoral e o bíceps femoral têm sido justificada pela menor velocidade durante o exercício aquático e, conseqüentemente, a menor resistência empregada ao deslocamento dos indivíduos ${ }^{(17-20,30,31)}$

Por outro lado, respostas neuromusculares diferentes podem ser esperadas quando a resistência ao exercício de caminhada em meio aquático é imposta com velocidades auto-selecionadas que resultam em velocidades de deslocamento horizontal similares às do meio terrestre (leve na terra vs alta na água) com deslocamento frontal livre ${ }^{(3,28,32)}$. Esses estudos têm sugerido atividade EMG semelhante ou até maior em músculos propulsores durante a caminhada em meio aquático. Recentemente, a possibilidade de gerar respostas neuromusculares aumentadas durante a caminhada aquática tem sido comprovada em exercícios realizados em esteira com velocidade fixa(17-20,30,31). Nessas análises, as comparações são realizadas com a mesma velocidade de caminhada entre os meios $(2,4 \mathrm{~km} / \mathrm{h})^{(17-20,30,31)}$. A atividade aumentada de músculos como o gastrocnêmio, o tibial anterior, vasto medial, o reto femoral e o bíceps femoral no meio aquático ocorreria devido ao aumento da resistência imposta ao movimento pela necessidade de vencer a maior densidade do fluído líquido comparado com a do ar em uma mesma velocidade de exercício ${ }^{(17-20,30,31)}$.

\section{Caminhada em piscina funda}

São recentes, e ainda em menor número, as análises neuromusculares comparativas entre a caminhada em terra e a caminhada em piscina funda. Kaneda et al. ${ }^{(33)}$ avaliaram as respostas neuromusculares de indivíduos caminhando com diferentes velocidades auto-selecionadas de esforço (leve, moderada e alta) e deslocamento frontal livre. Os resultados mostraram que em todas as velocidades auto-selecionadas houve maior atividade EMG dos músculos sóleo e gastrocnêmio durante a caminhada terrestre. Em contrapartida, o músculo bíceps femoral foi mais ativado durante a caminhada em piscina funda. Vale salientar que as velocidades de deslocamento horizontal não foram apresentadas, embora provavelmente tenham sido sempre menores no ambiente aquático. A menor atividade encontrada nos músculos gastrocnêmio e sóleo no exercício aquático foi justificada pela ausência de contato, e conseqüentes forças verticais, com o fundo durante a caminhada em piscina profunda. Assim, pode ser sugerido que esses músculos perdessem sua característica propulsora nessa forma de exercício aquático. Por outro lado, a maior atividade do músculo bíceps femoral foi atribuída a possível maior amplitude de movimento do 
quadril e do joelho na caminhada aquática. Essa maior amplitude de movimento da articulação do quadril durante a caminhada em piscina funda já havia sido registrada anteriormente ${ }^{(34)}$.

\section{RESPOSTAS CARDIORRESPIRATÓRIAS}

\section{Caminhada em piscina rasa}

As respostas cardiorrespiratórias durante a caminhada em piscina rasa estão bem descritas na literatura ${ }^{(25,35-38)}$. Tais respostas normalmente são investigadas durante o exercício executado com velocidade fixa e deslocamento reduzido (esteira). Nessas condições, as respostas cardiorrespiratórias ao exercício aquático são dependentes principalmente da profundidade de imersão dos indivíduos e da velocidade do exercício entre os meios.

Em relação à freqüência cardíaca $(F C)$, parece consensual que em exercícios de caminhada com velocidade fixa similar entre os meios e deslocamento reduzido (esteira), as respostas são maiores no meio aquático, independentemente de a profundidade de imersão ser no tornozelo, joelho, coxa ou na cintura dos indivíduos ${ }^{(39,40)}$. Essas respostas diferem das respostas encontradas após a imersão em repouso, onde bradicardia resultante do reflexo barorreceptor é esperada ${ }^{(10,16,41)}$. Esses achados sugerem que utilizando uma mesma velocidade de caminhada aquática e terrestre, a FC possa estar aumentada no meio aquático devido a maior exigência fisiológica necessária para mover o corpo na água, visto que a densidade desse fluído é muito superior à do $\operatorname{ar}^{(18,19)}$, tornando o exercício muito mais intenso nesse ambiente. Possivelmente, essa maior resposta da FC encontrada no meio aquático seria devida a algum mecanismo da imersão, que afetaria o sistema nervoso autônomo. Assim, maior retirada parassimpática poderia ocorrer em exercícios leves (como a caminhada,) o que justificaria esse grande aumento na resposta da $\mathrm{FC}^{(39,40)}$.

Porém, com os indivíduos imersos na profundidade do processo xifóide, respostas da FC semelhantes podem ser esperadas em velocidades moderadas e altas de exercício(24). Isso pode ser explicado por maior efeito da flutuação quando o corpo é imerso nessa profundidade. Com o efeito da flutuação aumentado sobre o indivíduo, a velocidade de deslocamento poderia ser reduzida em comparação com a velocidade da esteira, acarretando menor resistência oferecida ao exercício aquático. Esse fenômeno é comumente observado durante o exercício de corrida ${ }^{(40)}$. Ainda, as repostas da FC durante a caminhada em piscina rasa podem ser atenuadas com temperaturas da água mais frias (aproximadamente $28^{\circ} \mathrm{C}$ ) e aumentadas com temperaturas da água mais quentes (aproximadamente $36^{\circ} \mathrm{C}$ ) $(24,42)$.

Respostas semelhantes da FC também podem ser encontradas entre os dois ambientes utilizando diferentes velocidades do exercício de caminhada em esteira ${ }^{(14,30,31,36)}$. Nessa perspectiva, é necessário que a velocidade do exercício aquático seja exatamente a metade da velocidade do exercício realizado em terra com os indivíduos imersos ao nível do processo xifóide (terra $=2,4 \mathrm{~km} / \mathrm{h}, 3,6 \mathrm{~km} / \mathrm{h}$ e $4,8 \mathrm{~km} / \mathrm{h}$ vs água $=1,2 \mathrm{~km} / \mathrm{h}$, $1,8 \mathrm{~km} / \mathrm{h}$ e $2,4 \mathrm{~km} / \mathrm{h}$ ). Esse rigoroso controle da velocidade é possível em investigações que utilizam esteiras subaquáticas (Flowmil/) ${ }^{(30,31)}$. Todavia, nesses estudos a FC foi mensurada apenas durante um minuto de exercício. Esse pequeno tempo de coleta não parece o suficiente para estabilizar a FC dos indivíduos ${ }^{(40)}$. De qualquer forma, esses achados sugerem que as respostas da FC podem ser semelhantes entre os meios em comparações realizadas com diferentes velocidades de caminhada, uma vez que, nesse caso, o efeito da maior resistência proporcionada pela densidade do meio aquático seria suprido pela maior intensidade de esforço devido à maior velocidade do exercício em terra.

Em relação às respostas do consumo de oxigênio $\left(\mathrm{VO}_{2}\right)$, essas parecem semelhantes às encontradas para a FC. Comparando os indivíduos caminhando em esteira em ambos os meios com a mesma velocidade, a resposta do $\mathrm{VO}_{2}$ tem sido maior no meio aquático ${ }^{(24,35,40)}$. Essas respostas podem ser esperadas em diferentes profundidades (maléolo, patela, coxa e cicatriz umbilical) $)^{(35)}$ e temperaturas de imersão $\left(28 \text { e } 36^{\circ} \mathrm{C}\right)^{(24)}$. Contudo, essas respostas são encontradas apenas em velocidades moderadas e altas de caminhada (acima de 4,0km/h) ${ }^{(24)}$, uma vez que, em velocidades mais baixas, as respostas parecem semelhantes entre os meios ${ }^{(24,35)}$. Isso pode ser esperado devido ao fato de que em velocidades baixas de esforço durante a caminhada as forças de arrasto na água sejam minimizadas, reduzindo a resistência oferecida ao exercício $\mathrm{e}$ influenciando significativamente as respostas de $\mathrm{VO}_{2}{ }^{(17-20)}$. Embora $\mathrm{O}$ $\mathrm{VO}_{2}$ seja maior no meio aquático comparado com o meio terrestre nas primeiras condições citadas, o nível de imersão corporal pode interferir nessa magnitude ${ }^{(40)}$. $\mathrm{V} \mathrm{VO}_{2}$ no meio aquático durante a caminhada parece maior com os indivíduos imersos na profundidade da coxa quando comparados com a de indivíduos imersos na profundidade da cintura(40). Segundo os autores, a maior imersão dos indivíduos está relacionada com a maior redução no peso hidrostático. Esse fato acarretaria menor custo energético (menor consumo de oxigênio de músculos posturais) para sustentação do peso corporal em grandes profundidades de imersão.

Todavia, quando a comparação das respostas do $\mathrm{VO}_{2}$ é analisada em diferentes velocidades da caminhada entre os meios, essas são modificadas ${ }^{(37)}$. $\mathrm{O} \mathrm{VO}_{2}$ parece semelhante para as comparações em velocidades moderada e alta de esforço $(4,8 \mathrm{~km} / \mathrm{h}$ e $6,0 \mathrm{~km} / \mathrm{h}$ na terra vs 2,4km/h e 3,0km/h na água), mas pode apresentar menores valores no meio aquático em velocidades de esforço mais baixas $(3,6 \mathrm{~km} / \mathrm{h}$ na terra vs 1,8km/h na água). Novamente, a explicação para esses achados está relacionada com a maior resistência oferecida ao movimento pelo meio aquático devido a sua maior densidade, principalmente em velocidades moderada e alta de esforço.

\section{Caminhada em piscina funda}

Muitos estudos têm comparado as respostas cardiorrespiratórias máximas da corrida em piscina funda com a corrida em terra na esteira ${ }^{(1,43-48)}$. Apesar de estar claro que as respostas máximas de $\mathrm{VO}_{2} \mathrm{e}$ FC são mais baixas durante a corrida em piscina funda, essas respostas podem ser atenuadas em exercício submáximo ${ }^{(46,49-51)}$.

No que diz respeito à caminhada em piscina funda, as respostas cardiorrespiratórias analisadas parecem consensuais, embora sejam encontradas em pequeno número. Green et al. ${ }^{(52)}$, controlando a velocidade (leve, moderada e alta) da caminhada terrestre e aquática por cadências rítmicas similares entre os meios, e com deslocamento horizontal livre, encontraram respostas da FC sempre menores no meio aquático. Mais tarde, Robert et al. ${ }^{(53)}$, novamente, controlando a velocidade (moderada) da caminhada por uma cadência similar entre os meios e com deslocamento frontal livre, verificaram $\mathrm{FC} \mathrm{e} \mathrm{VO}_{2}$ menores durante o exercício aquático. Embora a velocidade de deslocamento horizontal dos indivíduos não tenha sido apresentada em nenhum desses estudos, possivelmente em uma mesma cadência de exercício esta é menor no meio aquático devido à resistência do fluído. Assim, a menor resposta da FC encontrada durante a caminhada em piscina funda é sugerida pela combinação de fatores como o aumento do retorno venoso devido ao efeito da pressão hidrostática sobre o corpo em imersão, e o conseqüente reflexo barorreceptor, e a menor resistência oferecida ao movimento devido à baixa velocidade de deslocamento. Já o menor $\mathrm{VO}_{2}$ observado pode ser atribuído também a esse último fato mencionado, além de os músculos antigravitacionais não serem necessários na água para suportar o peso corporal, devido à utilização do cinturão. Esse, provavelmente, é um fator preponderante para a diminuição no custo metabólico da caminhada na água quando comparada com a caminhada em meio terrestre ${ }^{(48)}$. 


\section{CONCLUSÃO}

Com a crescente popularização das atividades aquáticas e os registros de seus benefícios relacionados com a saúde de seus praticantes, modalidades como a caminhada em piscina rasa e a caminhada em piscina funda têm sido alvo de grande procura. Contudo, devido à dificuldade em controlar a intensidade dos exercícios no meio aquático, o conhecimento das respostas neuromusculares e cardiorrespiratórias da caminhada em diferentes situações nesse ambiente é de fundamental importância para profissionais da área.

As respostas neuromusculares e cardiorrespiratórias são muito dependentes da velocidade do exercício de caminhada em piscina rasa. A magnitude da atividade muscular, principalmente dos músculos propulsores, pode ser superior durante o exercício no ambiente aquático quando velocidades similares às do exercício terrestre são utilizadas. $\mathrm{O}$ mesmo pode ser esperado em relação à $\mathrm{FC}$ e ao $\mathrm{VO}_{2}$, indicando a possibilidade de utilizar esse exercício para o aumento do dispêndio

\section{REFERÊNCIAS BIBLIOGRÁFICAS}

1. Butts NK, Tucker M, Greening C. Physiologic responses to maximal treadmill and deep water running in men and women. Am J Sports Med 1991;19:612-4.

2. Kruel LFM. Alterações fisiológicas e biomecânicas em indivíduos praticando exercícios de hidroginástica dentro e fora d'água. Tese de Doutorado. Universidade Federal de Santa Maria, Santa Maria, 2000.

3. Miyoshi T, Shirota T, Yamamoto S, Nakazawa K, Akai M. Effect of the walking speed to the lower limb joint angular displacements, joint moments and ground reaction forces during walking in water. Disabil Rehabil 2004;26:724-32.

4. Alberton CL, Coertjens M, Figueiredo PAP, Kruel LFM. Behavior of oxygen uptake in water exercises performed at different cadences in and out of water. Med Sci Sports Exerc 2005;37:S103.

5. Taunton JE, Rhodes EC, Wolski LA, Donelly M, Warren J, Elliot J, et al. Effect of land-based and waterbased fitness programs on the cardiovascular fitness, strength and flexibility of woman aged 65-75 years. Gerontology 1996:42:204-10.

6. Oda S, Matsumoto T, Nagakawa K, Moriya K. Relaxation effects in humans of underwater exercise of moderate intensity. Eur J Appl Physiol 1999;80:253-9.

7. Pöyhönen T, Sipilä S, Keskinen KL, Hautala A, Savolainen J, Mälkiä E. Effects of aquatic resistance training on neuromuscular performance in healthy women. Med Sci Sports Exerc 2002;34:2103-9.

8. Takeshima N, Rogers ME, Watanabe WF, Brechue WF, Okada A, Yamada T, et al. Water-based exercise improves health-related aspects of fitness in older women. Med Sci Sports Exerc 2002;33:544-51.

9. Alves RV, Mota J, Costa MC, Alves JGB. Physical fitness and elderly health effects of hydrogymnastics. Rev Bras Med Esporte 2004;10:38-43.

10. Kruel LFM. Peso Hidrostático e Freqüência Cardíaca em Pessoas Submetidas a Diferentes Profundidades de Água. Dissertação de Mestrado. Universidade Federal de Santa Maria, Santa Maria, 1994

11. Kruel LFM, Tartaruga LAP. Estudo do percentual de redução no peso hidrostático através do método de imersão vertical em pessoas do sexo feminino em distintas faixas etárias. In: Congresso Brasileiro de Biomecânica, 9, 2001. Gramado. Anais. Porto Alegre: Escola de Educação Física da UFRGS, 2001. 1V., p. 186-91.

12. Barela AMF, Stolf SF, Duarte M. Biomechanical characteristics of adults walking in shallow water and on land. J Electromyogr Kinesiol 2006;16:250-6.

13. Barela AM, Duarte M. Biomechanical characteristics of elderly individuals walking on land and in water. J Electromyogr Kinesiol 2008;18:446-54.

14. Masumoto K, Takasugi S, Hotta N, Fujishima K, Iwamoto Y. Electromyigraphic analysis of walking in water in healthy humans. J Physiol Anthropol Appl Human Sci 2004;23:119-27.

15. Dowzer CN, Reilly T. Deep-water running. Sports Exercise and Injury 1998;4:56-61.

16. Reilly T, Dowzer CN, Cable NT. The physiology of deep water running. J Sports Sci 2003;21:959-72.

17. Pöyhönen T, Keskinen KL, Hautala A, Savolainen J, Mälkiä E. Human isometric force production and electromyogram activity of knee extensor muscles in water and on dry land. Eur J Appl Physiol 1999;80:52-6.

18. Pöyhönen T, Keskinen KL, Hautala A, Mälkiä E. Determination of hydrodinamic drag forces and drag coefficients on human leg/foot model during knee exercise. Clin Biomech 2000;15:256-60.

19. Pöyhönen T, Kyrolainen H, Keskinen KL, Hautala A, Savolainen J, Mälkiä E. Neuromuscular function during therapeutic knee exercise under water and on dry land. Arch Phys Med Rehabil 2001;82:1446-52.

20. Pöyhönen T, Kyrolainen $\mathrm{H}$, Keskinen $\mathrm{KL}$, Hautala A, Savolainen J, Mälkiä E. Electromyographic and kinematic analysis of therapeutic knee exercises under water. Clin Biomech 2001;16:496-504.

21. Ervilha UF, Duarte M. Amadio AC. Padrão do sinal eletromiográfico de músculos do membro inferior e cinemática do joelho durante o andar em ambiente aquático e terrestre. In: Congresso Brasileiro de Biomecânica, 9, 2001. Gramado. Anais. Porto Alegre: Escola de Educação Física da UFRGS, 2001. v.2, p.290-4.

22. Masumoto K, Takasugi S, Hotta N, Fujishima K, Iwamoto Y. Muscle activity and heart rate responses during backward walking in water and on dry land. Eur J Appl Physiol 2005;94:54-61.

23. Masumoto K, Takasugi S, Hotta N, Fujishima K, Iwamoto Y. A comparison of muscle activity and heart rate response during backward and forward walking on an underwater treadmill. Gait and Posture 2007; $25: 222-8$.

24. Hall J, Mcdonald IA, Maddison PJ, O'hare JP. Cardiorespiratory responses to underwater treadmill walking in healthy females. Eur J Appl Physiol 1998;77:278-84.

25. Shono T, Fujishima K, Hotta N, Ogaki T, Ueda T. Physiological responses to water-walking in middle aged women. J Physiol Anthropol 2001;20:119-23.

26. Alberton CL, Silva EM, Cadore EL, Coertjens M, Beyer PO, Marocco LF, et al. Electromiographic activity alterations during isometric actions performed in water and on dry land. Med Sci Sports Exerc 2007;39:S258.

27. Silva EM, Alberton CL, Tartaruga MP, Cadore EL, Becker ME, Brentano MA, et al. Electromiographic energético dos praticantes, sem sobrecarregar o sistema osteomuscular. Por sua vez, a atividade muscular mostra-se muito diferente da caminhada em meio terrestre durante a caminhada em piscina funda. Esse fato, resultante do diferente padrão de recrutamento muscular para a propulsão do movimento no exercício aquático, coloca a caminhada em piscina funda como uma alternativa de exercício para o fortalecimento, principalmente, de músculos do quadril. Já as respostas cardiorrespiratórias parecem sempre menores quando comparadas com as da caminhada em terra para uma mesma cadência de execução, devido à dificuldade em deslocar-se com velocidades semelhantes às do exercício terrestre. Apesar das diversas evidências apresentadas, alguns tópicos abordados ainda carecem de maior investigação para que melhores conclusões e recomendações possam ser estabelecidas.

Todos os autores declararam não haver qualquer potencial conflito de interesses referente a este artigo. signal reliability analysis during isometric and dynamic actions performed in different environments. Med Sci Sports Exerc 2007;39:5261

28. Miyoshi T, Shirota T, Yamamoto S, Nakazawa K, Akai M. Functional roles of lower-limb joint moments while walking in water. Clin Biomechanics 2005;20:194-201.

29. Miyoshi T, Nakazawa K, Tanizaki M, Sato T, Akai M. Altered Activation pattern in synergistic ankle plantarflexor muscles in a reduced-gravity environment. Gait and Posture 2006;94:99-201.

30. Shono T, Masumoto K, Fujishima K, Hotta N, Ogaki T, Adachi T. Gait Patterns and muscle activity in the lower extremities of elderly women during underwater treadmill walking against water flow. J Physiol Anthropol 2007;26:579-86.

31. Masumoto K, Shono T, Hotta N, Fujishima K. Muscle activation, cardiorespiratory response, and rating of perceived exertion in older subjects while walking in water and on dry land. J Electromyogr Kinesiol 2008;18:581-90.

32. Kato T, Sugagima Y, Koeda M, Fukuzawa S, Kitagawa K. Electromyogram activity of leg muscles during different types of underwater walking. Adv Exerc Sports Physiol 2002;8:39-44.

33. Kaneda K, Wakabayashi H, Sato D, Nomura T. Lower extremity muscle activity during different types and speeds of underwater movement. J Physiol Anthropol 2007;26:197-200.

34. Kilding AE, Scott MA, Mullineaux DR. A kinematic comparison of deep water running and overground running in endurance runners. J Strength Cond Res 2007;21:476-80.

35. Gleim GW, Nicholas J.A. Metabolic costs and heart rate responses to treadmill walking in water at different depths and temperatures. Am J Sports Med 1989;17:248-52.

36. Shono T, Fujishima K, Hotta N, Ogaki T, Ueda T, Otoki K, et al. Physiological responses and RPE during underwater treadmill walking in women of middle and advanced age. J Physiol Anthropol 2000;19:195-200.

37. Shono T, Fujishima K, Hotta N, Ogaki T, Masumoto K. Cardiorespiratory response to low-intensity walking in water and on land in elderly women. J Physiol Anthropol 2001;20:269-74.

38. Fujishima K, Shimizu T. Body temperature, oxygen uptake and heart rate during walking in water and on land at an exercise intensity based on RPE in elderly men. J Physiol Anthropol 2003;22:83-8.

39. Whitley JD, Schoene LL. Comparison of heart rate responses: water walking versus treadmill walking. Phys Ther 1987;67:1501-4

40. Pohl MB, Mcnaughton LR. The physiological responses to running and walking in water at different depths. Research in Sports Medicine 2003;11:63-78.

41. Coertjens M, Dias ABC, Silva RC, Rangel ACB, Tartaruga LAP, Kruel LFM. Determinação da bradicardia durante imersão vertical no meio líquido. In: Salão de Iniciação Científica, 12, 2000, Porto Alegre. Livro de Resumos. Porto Alegre: UFRGS, 2000. p. 341

42. Shimizu T, Kosaka M, Fujishima K. Human thermoregulatory responses during prolonged walking in water at 25, 30 and 35० C. J Appl Physiol 1998;78:473-8.

43. Gleim GW, Nicholas JA. Metabolic costs and heart rate responses to treadmill walking in water at different depths and temperatures. Am J Sports Med 1989;17:248-52.

44. Svedenha GJ, Seger J. Running on land and in water: comparative exercise physiology. Med Sci Sports Exerc 1992;24:1155-60.

45. Frangolias DD, Rhodes EC. Maximal and ventilatory threshold responses to treadmill and water immersion running. Med Sci Sports Exerc 1995;27:1007-13.

46. Glass B, Wilson D, Blessing D, Miller E. A physiological comparison of suspended deep water running to hard surface running. J. Strength Cond Res 1995;9:17-21.

47. Dowzer CN, Reilly T, Cable N, Nevill A. Maximal physiological responses to deep and shallow water running. Ergonomics 1999;42:275-81.

48. Nakanishi Y, Kimura T, Yoko Y. Maximal responses to deep water running at termoneutral temperature. Appl Human Sci 1999;18:31-5

49. Yamaji $K$, Greenley M, Northey DR, Hughsom RL. Oxygen uptake and heart rate responses to treadmill and water running. Can J Sport Sci 1990;15:96-8.

50. Demaere J, Ruby BC. Effects of deep water and treadmill running on oxygen uptake and energy expenditure in seasonally trained cross country runners. J Sports Med Phys Fitness 1997;37:175-81.

51. Mercer JA, Jensen RL. Heart rate at equivalent submaximal levels of $\mathrm{VO}_{2}$ do not differ between deep water running and treadmill running. J Strength Cond Res 1998;12:161-5.

52. Green JH, Cable NT, Elms H. Heart rate and oxygen consumption during walking on land. J Sports Med Phys Fitness 1990;30:49-52.

53. Robert JJ, Jones L, Bobo M. The physiologic response of exercising in the water and on land with and without the X1000 walk'n tone exercise belt. Res Quat Exerc Sport 1996;67:310-5. 